\title{
Estaciones automatizadas preanalíticas en el laboratorio de hemostasia. Estudio observacional descriptivo prospectivo, realizado en un hospital universitario de referencia entre el 15 de abril y 15 de julio de 2017
}

\author{
Luz Fernanda Sua ${ }^{1,2}$, María Alejandra Amezquita ${ }^{3}$, Daniel Esteban Hernández ${ }^{3}$, Mercedes Alcalá-Flores ${ }^{4}$, \\ Carl Sebastián Leib ${ }^{5}$, Marisol Aguirre-Rojas ${ }^{6,7}$, Liliana Fernández-Trujillo $0^{8,9}$
}

\begin{abstract}
RESUMEN
Introducción: los errores del proceso de análisis de las muestras del laboratorio clínico, impactan negativamente a la práctica médica, la seguridad del paciente e incrementan los costos de atención en la salud. El uso de estaciones automatizadas demostró una disminución de los errores en los laboratorios clínicos de inmunoquímica y hematología. Se propone comparar las primeras estaciones automatizadas de hemostasia con el método manual para determinar los interferentes en las muestras de cinco servicios del hospital durante tres meses.
\end{abstract}

Métodos: estudio observacional descriptivo prospectivo de corte trasversal. Las muestras que ingresaron al laboratorio clínico fueron analizadas por la estación automatizada y el método manual. Las interferentes bilirrubinas, hemoglobina, lipemia, volumen de llenado del tubo y obstrucción fluídica-coágulo se estudiaron con ambos métodos. Se realizó el análisis estadístico y se calculó el índice kappa para determinar la fuerza de la concordancia entre los métodos.

Resultados: de 8.970 muestras analizadas, 29 \% provinieron del servicio de urgencias. Las muestras aportadas por la unidad de cuidado intensivo reportaron más interferentes con

\footnotetext{
Patóloga, MD, PhD. Departamento de Patología y Medicina de Laboratorio, Fundación Valle de Lilli, Cali, Colombia.

Profesora Clínica Asistente, MD, PhD. Facultad de Ciencias de la Salud, Universidad Icesi, Cali, Colombia.

Médicos Internos, Facultad de Ciencias de la Salud, Universidad Icesi, Cali, Colombia.

Bacterióloga. Departamento de Patología y Medicina de Laboratorio, Fundación Valle de Lilli, Cali, Colombia.

Médico Asistente en Investigación. Centro de Investigaciones Clínicas, Fundación Valle del Lili, Cali, Colombia.

Asesora Metodológica, MD, MPH. Centro de Investigaciones Clínicas, Fundación Valle del Lili, Cali, Colombia.

Profesora hora cátedra. Departamento Salud Pública y Medicina Comunitaria, Facultad de Ciencias de la Salud, Universidad Icesi, Cali, Colombia.

Jefe Departamento de Medicina Interna, Servicio de Neumología y Neumología Intervencionista, Fundación Valle del Lili, Cali, Colombia.

Profesora Clínica Asociada, Facultad de Ciencias de la Salud, Universidad Icesi, Cali, Colombia.
}

Correspondencia: Luz Fernanda Sua; lufer24@hotmail.com

Recibido: julio 24 de 2018

Aceptado: enero 15 de 2019

Cómo citar: Sua LF, Amézquita MA, Hernández DE, Alcalá-Flores M, Leib CS, Aguirre-Rojas M, et al. Estaciones automatizadas preanalíticas en el Laboratorio de hemostasia. Estudio observacional descriptivo prospectivo, realizado en un hospital universitario de referencia entre el 15 de abril y 15 de julio de 2017. latreia. 2019 Jul-Sep;32(3):177-183. D0I 10.17533/udea.iatreia.12. 
ambos métodos; la estación automatizada reportó más interferentes que el método manual, con un índice kappa 0,52; la bilirrubina fue el interferente más detectado por ambos métodos. El método manual no evidenció el interferente volumen de llenado del tubo ni obstrucción fluídica-coágulo, los cuales fueron detectados por la estación automatizada.

Conclusiones: la estación automatizada detectó más interferentes en comparación con el método manual. Los interferentes son errores preanalíticos en el laboratorio de hemostasia y son detectados con poca frecuencia de forma visual, especialmente los interferentes volúmenes de llenado del tubo y obstrucción fluídica-coágulo.

\section{PALABRAS CLAVE}

Control de Calidad; Fase Preanalítica; Hemostasia

\section{SUMMARY}

Automated preanalytical units in the hemostasis laboratory: an observational descriptive prospective study developed in a university hospital between April 15 to July 15, 2017

Introduction: Errors in the process of analyzing a clinical laboratory sample have a negative impact on medical practice, patient safety and health care cost. The use of automated units demonstrated the reduction of errors in the clinical laboratories of immunochemistry and hematology. The purpose of the study is to compare the first automated unit with a manual method and to determine the interferences informed in the samples provided by five services studied in the hospital for three months.

Methods: A prospective descriptive study was developed. The automated unit and manual method analyzed all analytical requested samples received by the clinical laboratory. Interferences such as bilirubin, hemoglobin, lipemic, tube filling volume, and fluidclot obstruction were studied with both methods. The statistical analysis was performed and the kappa coefficient was calculated to determine the strength of agreement between both methods.
Results: Of the 8.970 samples analyzed, $29 \%$ came from the emergency service. The samples contributed by the intensive united care reported more interferences with both methods. The automated unit reported more interference than the manual method, with a kappa coefficient of 0,52 . Bilirubin was the most commonly detected interference by both methods. The manual method did not show the interferences volume of tube filling or fluidic obstruction-clot, which have been detected by the automated unit.

Conclusions: Automated unit detected more interference in comparison with the manual method. The interferences are preanalytical errors in the hemostasis laboratory and are less frequently detected by $\nabla$ isual examination, especially interferences volume of tube filling or fluidic obstruction-clot.

\section{KEY WORDS}

Hemostasis; Pre-Analytical Phase; Quality ControI

\section{INTRODUCCIÓN}

El proceso analítico de una muestra comprende las fases preanalítica, analítica y postanalítica (1). Durante la fase preanalítica ocurren del 46 al $68 \%$ de los errores del proceso de análisis de una muestra de laboratorio clínico (2). En la mayoría de los casos, los resultados de estas muestras son considerados índices de "diagnóstico» (3), por lo tanto, es de gran importancia intervenir estos errores preanalíticos. Se ha reportado que el uso de procesos automatizados totales, modulares o estaciones como soporte de las actividades preanalíticas, han logrado minimizar los errores en esta fase (4), específicamente en las muestras de inmunoquímica y hematología.

Los errores médicos en la práctica clínica causan de 50.000 a 100.000 muertes prevenibles cada año, y es la octava causa más común de muerte en los Estados Unidos (5). Se ha reportado que un 70 a $80 \%$ de las decisiones médicas se basan en los reportes de laboratorio, por lo cual, la calidad de estos es de gran importancia (6). Gracias al desarrollo de la ciencia, la implementación de la informática y la tecnología se 
ha logrado mejorar el desempeño del proceso analítico y postanalítico, siempre que las buenas prácticas del control de calidad se cumplan (7).

Los errores de la fase preanalítica pueden aparecer desde la solicitud de la prueba por parte del médico, hasta el momento en que la muestra está lista para el análisis en el laboratorio (6). Se ha visto que el $26 \%$ de los errores durante esta fase resultan en efectos perjudiciales para el paciente (8) y, además, aportan al $75 \%$ de los gastos de una institución hospitalaria (9).

En los últimos 25 años se ha invertido en el desarrollo de múltiples procesadores automatizados. En la actualidad son exitosamente utilizados en laboratorios clínicos que procesan un alto volumen de muestras (10). Más de 2.000 laboratorios alrededor del mundo usan procesos automatizados totales, modulares o estaciones como soporte de las actividades preanalíticas, especialmente diseñados para pruebas de inmunoquímica y hematología $(10,11)$.

En el Laboratorio de hemostasia, en particular, la toma de la muestra, el llenado del tubo, la relación del anticoagulante y la muestra, así como la agitación del tubo, son una condición crítica, ya que un interferente como Ia bilirrubina, la hemoglobina, la lipemia, el volumen de llenado del tubo y la obstrucción fluídica-coágulo, pueden generar la prolongación o acortamiento de las pruebas de coagulación, reportar niveles inadecuados de pruebas como fibrinógeno, dímero D o factores de coagulación; lo que genera falsos positivos o falsos negativos que pueden desencadenar diagnósticos erróneos y gastos innecesarios (12).

Tradicionalmente, los laboratorios de hemostasia no cuentan con estaciones automatizadas y se han establecido criterios para la aceptación $y$ el rechazo de las muestras de forma visual. El proceso de análisis es manual, lo que lo hace susceptible a la subjetividad del ojo humano que implica errores, pérdida de tiempo y aumento de costos (7). Por lo tanto, los métodos automatizados actualmente desarrollados desde el año 2016 y aprobados por la FDA (Administración de Alimentos y Medicamentos de los Estados Unidos), permiten evitar el error humano en la fase preanalítica, mejorando el análisis y optimizando el manejo de las muestras $(13,14)$.

El propósito de este estudio es comparar las primeras estaciones automatizadas introducidas en Colombia y en la Institución (equipos ACL-TOP350, Intrumentation Laboratory a Werfen Company) con la evaluación visual de las muestras de hemostasia (método manual) realizada por los analistas clínicos y determinar los interferentes reportados por cada uno de los 5 servicios estudiados en el hospital, entre el 15 de abril y el 15 de julio de 2017.

\section{MATERIALES Y MÉTODOS}

\section{Consideraciones éticas}

Esta investigación fue aprobada por el Comité de Ética en Investigación Biomédica. Se acogió a la normativa internacional de investigación biomédica y nacional de Colombia consignada en la resolución 8430 de 1993, en la cual, según el artículo 11 numeral a, esta es una investigación sin riesgo, pues no involucró directamente a los pacientes y no se intervinieron médicamente. Las muestras para el análisis de laboratorio clínico utilizadas en el estudio fueron tomadas rutinariamente a los pacientes como parte del proceso de atención en salud. EI estudio no modificó el diagnóstico ni el pronóstico de los pacientes.

\section{Población de estudio}

La muestra del estudio fue a conveniencia, muestreo no probabilístico. Todas las muestras que ingresaron para el análisis de laboratorio clínico entre el 15 de abril y el 15 de julio de 2017 fueron incluidas en el estudio. Las pruebas incluidas para el estudio fueron TP, TTPa, fibrinógeno, dímero $\mathrm{D}$, factores de la coagulación, proteína $\mathrm{S}$, proteína $\mathrm{C}$ y anticoagulante lúpico. Se incluyeron los 5 servicios del hospital (Cirugía, Hospitalización, Laboratorio clínico ambulatorio, Unidad de cuidado intensivo [UCI] y Urgencias) que solicitaron con mayor frecuencia este tipo de pruebas de hemostasia.

\section{Diseño del estudio}

Estudio observacional descriptivo prospectivo de corte trasversal con alcance comparativo, desde abril 15 a julio 15 de 2017, periodo en el cual se implementó por primera vez en la Institución la estación automatizada para la fase preanalítica del Laboratorio de hemostasia. Todas las muestras fueron analizadas por la 
estación automatizada y el método manual. En este estudio se compararon los interferentes detectados por la estación automatizada y los 14 profesionales de laboratorio clínico (método manual), entre los cuales se contaba con bacteriólogos y laboratoristas clínicos con experiencia entre 5 y 20 años. Los datos fueron consignados en el sistema digital del Laboratorio de hemostasia para el reporte de los interferentes de bilirrubina, hemoglobina, lipemia, volumen de llenado del tubo (nivel de muestra adecuado $>90 \%$ del volumen) y obstrucción fluídica-coágulo.

\section{Método estadístico}

Posterior a la validación de la calidad de la base de datos, se realizó el análisis estadístico de los resultados reportados por la estación automatizada y el método manual y se calculó el índice kappa para determinar la fuerza de concordancia entre ambos métodos. Los análisis estadísticos se realizaron utilizando Stata ${ }^{\circledR}$, versión 14.

\section{RESULTADOS}

En el periodo del estudio, se analizaron un total de 8.970 muestras provenientes de los 5 servicios del hospital, el servicio de Urgencias fue el que más muestras aportó con un 29 \% del total (Tabla 1). La UCI reportó el mayor número de interferentes con un $36 \%$, seguido por Hospitalización con $26 \%$, Urgencias con $21 \%$, Cirugía con 10 \% y Laboratorio clínico ambulatorio con 8 \% (Tabla 2). Con el método manual, el servicio que reportó más interferentes fue la UCI: $53 \%$, seguida por Hospitalización: 31 \%, Urgencias: 16 \% y Cirugía: $1 \%$. No se detectaron interferentes en las muestras procedentes del laboratorio clínico ambulatorio con el método manual (Tabla 3). La estación automatizada detectó 421 interferentes y el método manual 154, con un índice kappa 0,52. Los interferentes informados por Ia estación automatizada fueron bilirrubina en un $56 \%$, volumen de llenado del tubo en un $26 \%$, obstrucción fluídica-coágulo en un $12 \%$, hemoglobina en un $5 \%$ y lipemia en un $1 \%$ de las muestras analizadas (Tabla 4). Con el método manual los interferentes detectados fueron la bilirrubina en un $90 \%$, hemoglobina en $6 \%$ y lipemia en $4 \%$ de las muestras. Con este método no se reportó interferente de volumen de llenado del tubo ni obstrucción fluídica-coágulo (Tabla 4).
Tabla 1. Total de muestras aportadas por los servicios del hospital

\begin{tabular}{ccc}
\hline Servicios & $\mathbf{n}$ & $\%$ \\
\hline Cirugía & 139 & 2 \\
\hline Hospitalización & 2.303 & 26 \\
\hline Laboratorio clínico ambulatorio & 1.639 & 18 \\
\hline UCI & 2.252 & 25 \\
\hline Urgencias & 2.637 & 29 \\
\hline Total general & 8.970 & 100 \\
\hline
\end{tabular}

Tabla 2. Interferentes reportados por los servicios con la estación automatizada

\begin{tabular}{|ccc|}
\hline Muestras con interferentes (estación automatizada) \\
\hline Servicios & $\mathbf{n}$ & $\%$ \\
\hline Sin interferente & 8.549 & 95 \\
\hline Interferente & 421 & 5 \\
\hline Cirugía & 41 & 10 \\
\hline Hospitalización & 109 & 26 \\
\hline Laboratorio clínico ambulatorio & 32 & 8 \\
\hline UCl & 150 & 36 \\
\hline Urgencias & 89 & 21 \\
\hline
\end{tabular}

Tabla 3. Interferentes reportados por los servicios con el método manual

\begin{tabular}{|ccc|}
\hline \multicolumn{3}{c}{ Muestras con interferentes (método manual) } \\
\hline Servicios & $\mathbf{n}$ & $\%$ \\
\hline Sin interferente & 8.816 & 98 \\
\hline Interferente & 154 & 2 \\
\hline Cirugía & 1 & 1 \\
\hline Hospitalización & 47 & 31 \\
\hline Laboratorio clínico ambulatorio & 0 & 0 \\
\hline UCl & 82 & 53 \\
\hline Urgencias & 24 & 16 \\
\hline
\end{tabular}


Tabla 4. Interferentes reportados por la estación automatizada y el método manual

\begin{tabular}{ccc}
\hline Muestras con interferente (estación automatizada) \\
\hline Tipo de interferente & $\mathbf{n}$ & $\%$ \\
\hline Sin interferente & 8.549 & 95 \\
\hline Interferente & 421 & 5 \\
\hline Bilirrubina & 234 & 56 \\
\hline Hemoglobina & 19 & 5 \\
\hline Lipemia & 6 & 1 \\
\hline Volumen de llenado del tubo & 110 & 26 \\
\hline Obstrucción fluídica- coágulo & 52 & 12 \\
\hline Muestras con interferente (método manual) \\
\hline Tipo de interferente & $\mathbf{n}$ & $\%$ \\
\hline Sin interferente & 8.816 & 98 \\
\hline Interferente & 154 & 2 \\
\hline Bilirrubina & 139 & 90 \\
\hline Hemoglobina & 9 & 6 \\
\hline Lipemia & 6 & 4 \\
\hline Volumen de & 0 & 0 \\
\hline Obstrucción fluídica- coágulo & 0 & 0 \\
\hline
\end{tabular}

\section{DISCUSIÓN}

Los hallazoos de este estudio permiten determinar lo importante que es para los laboratorios de hemostasia avanzar hacia la automatización de la fase preanalítica, ya que la inspección visual no es suficiente para determinar los interferentes de las pruebas de hemostasia, predominando las directamente relacionadas con la toma de la muestra, volumen de llenado del tubo y obstrucción fluídica-coágulo. Se han aplicado métodos modernos en el laboratorio clínico para reducir los errores en las fases preanalítica, analítica y postanalítica del procesamiento de las muestras (15). Sin embargo, los interferentes se encuentran más comúnmente en las fases preanalítica y postanalítica que en la analítica. La principal causa del problema es que algunas fases no tienen control directo del personal del laboratorio (16) y, la fase preanalítica no lo está, por ello es importante contar con un sistema automatizado de calidad para las muestras. Los errores preanalíticos son causados principalmente por humanos, y la mayoría de estos errores se pueden prevenir (17-19). Esto es comprensible, ya que la fase preanalítica implica un manejo mucho más humano en comparación con las fases analítica y postanalítica (20).

Debemos considerar que existen interferentes modificables que son secundarios a la toma de las muestras. Los interferentes de volumen de llenado del tubo y obstrucción fluídica-coágulo están asociados a la técnica de la flebotomía que podría presentarse en pacientes críticamente enfermos, como los encontrados en los servicios de Urogencias, Hospitalización y UCI, estos presentaron muestras con más interferentes. EI personal asignado para la toma de muestra en estos servicios es frecuentemente rotado según la distribución de turnos, generando variedad del personal involucrado en los procesos de las pruebas $(21,22)$. Por lo tanto, el personal de enfermería y de laboratorio debe ser el más especializado en tomar muestras y en conocer las indicaciones de cada prueba de hemostasia, lo que se evidenció en este estudio, en donde se encontró menos interferentes en las muestras provenientes del laboratorio clínico ambulatorio.

Los interferentes encontrados con mayor frecuencia por ambos métodos fueron la bilirrubina, hemoglobina y lipemia, asociados a la condición clínica del paciente. La hemólisis puede tener un causal compartido entre la condición clínica del paciente y la toma de la muestra cuando se fuerza la sangre a través de la aguja o se sacuden vigorosamente los tubos (18).

La detección manual de muestras hemolizadas en laboratorios de hemostasia es más difícil que en los laboratorios de hematología y bioquímica, lo que puede genera una frecuencia falsamente menor de error preanalítico (23), lo cual se puede observar en este estudio, la estación automatizada detectó más interferentes que el método manual. Los errores preanalíticos pueden impactar en las decisiones médicas, las cuales se basan en los reportes de laboratorio. Lo que conlleva a solicitar pruebas complementarias de extensión innecesarias y tratamientos no justificados que son costosos y pueden comprometer la seguridad del paciente.

Es importante promover la identificación del interferente, esto siognifica que debe existir en la organización del laboratorio clínico y de hemostasia una cultura 
que tienda a la detección del interferente, contar con indicadores cuyo análisis periódico y sistemático sea el motor e inicio de la mejora continua en la toma de las muestras.

Se encontraron como limitantes del estudio que las actividades preanalíticas para la hemostasia no se encuentran automatizadas y los indicadores de calidad en la toma de las muestras aún no se han armonizado mundialmente; se le suma el método descriptivo no probabilístico, el cual no tiene el suficiente poder estadístico para determinar la sensibilidad y especificidad de la estación automatizada comparada con el método manual. Se proponen futuros estudios para garantizar altos estándares de calidad en los laboratorios clínicos de hemostasia.

\section{CONCLUSIONES}

La implementación de la estación automatizada detectó más interferentes en comparación con el método manual. Los interferentes son errores preanalíticos en el Laboratorio de hemostasia y son detectados con poca frecuencia de forma visual, especialmente el interferente volumen de llenado del tubo y obstrucción fluídicacoágulo. El uso de estaciones preanalíticas automatizadas se traduce en una mayor eficiencia con alta calidad en el laboratorio de hemostasia, mejor atención del paciente y una reducción de los costos hospitalarios. Desafortunadamente, las actividades preanalíticas, la gestión de muestras inadecuadas y las políticas de presentación de informes de interferentes no están totalmente estandarizadas ni armonizadas en todo el mundo.

\section{CONFLICTOS DE INTERESES}

Ninguno por declarar.

\section{REFERENCIAS BIBLIOGRÁFICAS}

1. Laposata M. Concepts in Laboratory Medicine. In: Laposata $M$, editor. Laboratory Medicine: The Diagnosis of Disease in the Clinical Laboratory. New York, NY: McGraw-Hill Education; 2014.

2. Najat D. Prevalence of Pre-Analytical Errors in Clinical Chemistry Diagnostic Labs in Sulaimani City of
Iraqi Kurdistan. PLoS One. 2017 Jan;12(1):e0170211. DOI 10.1371/journal.pone.0170211.

3. Plebani $M$. The detection and prevention of errors in laboratory medicine. Ann Clin Biochem. 2010 Mar;47(Pt 2):101-10. DOI 10.1258/acb.2009.009222.

4. Lippi G, Ippolito L, Favaloro EJ. Technical evaluation of the novel preanalytical module on instrumentation laboratory ACL TOP: advancing automation in hemostasis testing. J Lab Autom. 2013 Oct; 18(5):38290. DOI 10.1177/2211068213491747.

5. Young DS, Sachais BS, Jefferies LC. Laboratory costs in the context of disease. Clin Chem. 2000 Jul;46(7):967-75.

6. Cano Corres R, Fuentes Arderiu X. Errores en el laboratorio clínico. Laboratori Clínic [Internet]. Disponible en: http://www.ifcc.org/media/214854/Errores\%20 en\%20el\%20laboratorio\%20c1\%C3\%ADnico.pdf

7. HarsimranKaur VN, Selhi PK, Sood N, Singh A. Preanalytical Errors in Hematology Laboratoryan Avoidable Incompetence. Iran J Pathol. 2016 Spring; 11(2):151-4.

8. Donaldson MS, Corrigan JM, Kohn LT, editors. To err is human: building a safer health system. Vol. 6. Washington D. C.: National Academies Press; 2000.

9. Green SF. The cost of poor blood specimen quality and errors in preanalytical processes. Clin Biochem. 2013 Sep;46(13-14):1175-9. DOI 10.1016/j.clinbiochem.2013.06.001.

10. Da Rin G. Pre-analytical workstations: a tool for reducing laboratory errors. Clin Chim Acta. 2009 Jun;404(1):68-74. DOI 10.1016/j.cca.2009.03.024.

11. Felder RA, Kost GJ. Automation. Part 1. Modular stepwise automation and the future of diagnostic testing. MLO Med Lab Obs. 1998 Apr;30(4):22-7.

12. Nagant $C$, Rozen L, Demulder A. HIL Interferences on Three Hemostasis Analyzers and Contribution of a Preanalytical Module for Routine Coagulation Assays. Clin Lab. 2016 Oct;62(10):1979-87. DOI 10.7754/ Clin.Lab.2016.160313.

13. Favaloro EJ, Funk DM, Lippi GL. Pre-analytical variables in coagulation testing associated with diagnostic errors in hemostasis. Lab Med. 2012 Feb;43(2):1-10. DOI 10.1309/LM749BQETKYPYPVM.

14. Fidler JR. Task analysis revisited: refining the phlebotomy technician scope of practice and assessing 
Iongitudinal change in competencies. Eval Health Prof. 2007 Jun;30(2):150-69.

15. Plebani M. Errors in clinical laboratories or errors in laboratory medicine? Clin Chem Lab Med. 2006;44(6):750-9.

16. Mohammedsaleh ZM, Mohammedsaleh F. A review article of the reduce errors in medical laboratories. Glob J Health Sci. 2014 Jul;7(1):46-51. DOI 10.5539/ gjhs. $77 \mathrm{n} 1 \mathrm{p} 46$.

17. Carraro P, Plebani M. Errors in a stat laboratory: types and frequencies 10 years later. Clin Chem. 2007 Jul;53(7):1338-42.

18. Kalra J. Medical errors: impact on clinical laboratories and other critical areas. Clin Biochem. 2004 Dec;37(12):1052-62.

19. Astion ML, Shojania KG, Hamill TR, Kim S, Ng VL. Classifying laboratory incident reports to identify problems that jeopardize patient safety. Am J Clin Pathol. 2003 Jul;120(1):18-26.
20. Rynning M, Wentzel-Larsen T, Bolann BJ. A model for an uncertainty budget for preanalytical variables in clinical chemistry analyses. Clin Chem. 2007 JuI; 53(7):1343-8.

21. Abdollahi A, Saffar H, Saffar H. Types and Frequency of Errors during Different Phases of Testing At a Clinical Medical Laboratory of a Teaching Hospital in Tehran, Iran. N Am J Med Sci. 2014 May;6(5):224-8. DOI 10.4103/1947-2714.132941.

22. Ashavaid TF, Dandekar SP, Khodaiji S, Ansari MH, Singh AP. Influence of method of specimen collection on various preanalytical sample quality indicators in EDTA blood collected for cell counting. Indian J Clin Biochem. 2009 Oct;24(4):356-60. DOI 10.1007/s12291009-0064-4.

23. Chawla R, Goswami $\nabla$, Tayal D, Mallika $\nabla$. Identification of the types of preanalytical errors in the clinical chemistry laboratory: 1-year study of G.B. Pant Hospital. Lab Med. 2010 Feb;41(2):89-92. DOI 10.1309/ LM9JXZBMLSVJT9RK. 